This is a postprint of an article published in Faix, J., Breitsprecher, D., Stradal, T.E.B., Rottner, K.

Filopodia: Complex models for simple rods

(2009) International Journal of Biochemistry and Cell Biology, 41 (8-9), pp. 1656-1664. 


\title{
Filopodia: complex models for simple rods
}

\author{
1,*Jan Faix, ${ }^{1}$ Dennis Breitsprecher, ${ }^{2}$ Theresia E.B. Stradal, and ${ }^{3}$ Klemens Rottner
}

${ }^{\mathbf{1}}$ Institute for Biophysical Chemistry, Hannover Medical School, Carl-Neubergstr. 1, D-30625

Hannover, Germany; ${ }^{2}$ Signalling and Motility Group and ${ }^{3}$ Cytoskeleton Dynamics Group,

Helmholtz Centre for Infection Research (HZI), Inhoffen Strasse 7, D-38124 Braunschweig, Germany.

*To whom correspondence should be addressed:

Email: faix@bpc.mh-hannover.de

phone: +49-511-532-2928

Fax: $\quad+49-511-532-5966$

Key words: actin, Arp2/3-complex, Ena/VASP, formin, IRSp53 


\begin{abstract}
Filopodia are prominent cell surface protrusions filled with bundles of linear actin filaments that drive their protrusion. These structures are considered important sensory organelles, for instance in neuronal growth cones or during the fusion of sheets of epithelial tissues. In addition, they can serve a precursor function in adhesion site or stress fibre formation. Actin filament assembly is essential for filopodia formation and turnover, yet the precise molecular mechanisms of filament nucleation and/or elongation are controversial. Indeed, conflicting reports on the molecular requirements of filopodia initiation have prompted researchers to propose different types and/or alternative or redundant mechanisms mediating this process. However, recent data shed new light on these questions, and they indicate that the balance of a limited set of biochemical activities can determine the structural outcome of a given filopodium. Here we focus on discussing our current view of the relevance of these activities, and attempt to propose a molecular mechanism of filopodia assembly based on a single core machinery.
\end{abstract}




\section{Introduction}

In order to move, individual cells and tissues have to be able to change their shape, and to exert force onto surrounding cells and substrata (Pollard and Borisy, 2003, Small et al., 2002). Force generation is mediated by different types of motors, such as myosins stepping or gliding along actin filaments, but also by the assembly of actin monomers into filaments. The latter actin polymerisation events can mediate pushing forces that are exploited - for instance - by intracellular pathogens that propel through the cytoplasm of infected cells (Lambrechts et al., 2008, Stevens et al., 2006). However, actin polymerisation also powers the forward movement of different types of cellular protrusions, such as lamellipodia and filopodia. Lamellipodia are thin, membrane-bound sheets of cytoplasm, comprised of dense actin filament networks, generated by the nucleation of actin filaments at the anterior networkmembrane interface (Lai et al., 2008, Wang, 1985). When protruding in three dimensions or after lifting up from the two-dimensional substrate to give rise to curled membranes, they are usually referred to as ruffles (Small et al., 2002).

Many cell types express additional types of protrusions, rod-like, highly dynamic structures termed filopodia, which contain relatively stiff bundles of actin filaments, yet can bend over, and surf about the cell surface while they protrude (Faix and Rottner, 2006, Ladwein and Rottner, 2008, Small et al., 2002). Average filopodia are estimated to contain a few dozens of filaments that fill plasma membrane rods of $100-300 \mathrm{~nm}$ in diameter.

In addition to filopodia that are able to protrude independently of the rest of the cell periphery, some cell types on top of that, or exclusively, contain bundles of actin filaments that are almost entirely embedded into the lamellipodium, and most commonly called microspikes (Mattila and Lappalainen, 2008, Small et al., 2002), although those have not stringently been separated from filopodia in the literature (Svitkina et al., 2003). Microspikes or structures sometimes also referred to as ribs (Adams, 2001), are frequently tilted relative to the direction 
of lamellipodium protrusion, causing a prominent lateral movement within the lamellipodium that does not require any additional motile mechanism than actin polymerisation at their tips (Oldenbourg et al., 2000). Although microspikes can develop into filopodia and vice versa, the two of them may still be considered distinct entities, since they display large differences in their dynamics and presumably regulation, last not least because microspikes per definition require the presence of a lamellipodium, whereas filopodia do not (Nicholson-Dykstra and Higgs, 2008, Steffen et al., 2006).

The protrusion of filopodia is also powered by actin polymerisation at their tips (Mallavarapu and Mitchison, 1999), although the origin of these polymerising filaments and their mechanism of nucleation is controversial (see also below). The occurrence of filopodia in different cellular systems or eukaryotic species, and the signalling pathways inducing them have been extensively discussed in comprehensive, recent reviews (Faix and Rottner, 2006, Gupton and Gertler, 2007, Mattila and Lappalainen, 2008, Mellor, 2009; see also Figure 1). We will thus put more emphasis here on the discussion of recent progress in our understanding of potential mechanisms of filament generation and elongation in filopodia, as exemplified by the biochemical activities mediated by members of the formin and Ena/VASP families.

\section{Rho-GTPases}

Small GTPases of the Rho-family belong to the most commonly used 'inducers' of filopodia formation. Seminal studies by Hall and others had originally demonstrated that a constitutively active variant of mammalian Cdc42 induces mixtures of lamellipodia and filopodia or filopodia alone if lamellipodia protrusion is simultaneously suppressed by dominant negative Rac (Kozma et al., 1995, Nobes and Hall, 1995). These observations thus already provided evidence for separable pathways of lamellipodia and filopodia protrusion 
(see also below). The GTPase cycle of Rho-GTPases is regulated by large protein families catalysing either the GTPase reaction (GTPase activating proteins, GAPs,) or GTP reloading (guanine nucleotide exchange factors, GEFs) (Moon and Zheng, 2003, Rossman et al., 2005). In addition, Rho GTPases are targeted to and activated at the plasma or intracellular membranes, which involves posttranslational modifications and is prevented by guanine nucleotide dissociating inhibitors (GDIs) mediating sequestration of inactive GTPases in the cytosol (DerMardirossian and Bokoch, 2005). Since Rho-GTPases signal to the cytoskeleton through binding to effector proteins in their active, GTP-loaded form, the current model of induction of a filopodium involves the targeting to and GTP-loading of the GTPase at or near the plasma membrane, and subsequent induction of actin assembly to drive forward filopodium protrusion (Disanza et al., 2005). It had appeared intuitive therefore to assume that each Cdc42 effector established to function in actin assembly will also engage in the induction of actin-dependent filopodia in cells. However, more recent observations indicated that this assumption was preliminary (Faix and Rottner, 2006). For instance, neither NWASP, a direct Cdc42-effector and activator of the actin nucleating machine Arp2/3-complex (Stradal et al., 2004) nor Arp2/3-complex itself turned out to be essential for filopodia formation (Gomez et al., 2007, Lommel et al., 2001, Nicholson-Dykstra and Higgs, 2008, Sarmiento et al., 2008, Steffen et al., 2006). However, conflicting studies reported filopodia reduction (but not removal) upon Arp2/3-complex silencing (Korobova and Svitkina, 2008) or N-WASP deletion (Snapper et al., 2001). These factors were therefore concluded to contribute to filopodia or microspike formation, at least indirectly, as both N-WASP and Arp2/3complex do not accumulate in these structures (Ladwein and Rottner, 2008, Svitkina and Borisy, 1999, Svitkina et al., 2003). Although clarification of this issue awaits further study, we favour a negligible role of the N-WASP/Arp2/3-complex pathway in filopodia formation. Finally, recent data highlighted other factors such as formins or IRSp53 family members to likely operate in these processes (see below). Additional difficulties to sketch resilient 
signalling pathways of filopodium formation come from the fact that Cdc42 is not essential for this process, at least not in every cell type (Czuchra et al., 2005, Yang et al., 2006), and that several out of the 20 mammalian Rho-GTPases are capable of inducing these structures, e.g. Cdc42-subfamily members such as Wrch1 (Aspenstrom et al., 2004, Ruusala and Aspenstrom, 2008), or RhoD and Rif (Ellis and Mellor, 2000, Pellegrin and Mellor, 2005). In

Dictyostelium, filopodia can be induced by at least four GTPases, Rac1A, B, C, and G (Dumontier et al., 2000, Somesh et al., 2006), but not all Rac GTPases expressed in this organism have been thoroughly examined. Clearly, future efforts to improve our knowledge on the commonalities and specificities of signalling pathways driving filopodia formation will have to include attempts to abolish these structures by interference with multiple GTPases simultaneously.

\section{Models for filament generation in filopodia: convergent elongation versus nucleation}

B16-F1 melanoma cells migrating on laminin have developed into a prominent model for the analysis of molecular events accompanying actin-based protrusion (Ballestrem et al., 1998, Lai et al., 2008, Rottner et al., 1999, Small et al., 2002, Svitkina et al., 2003). The lamellipodia of these cells are explicitly rich in microspikes that frequently adopt lateral motility, and show fusing or even crossing behaviour (Koestler et al., 2008, Svitkina et al., 2003, Vignjevic et al., 2006). Based on the dynamics of these microspikes visualised by livecell imaging and on correlative electron microscopy studies, Svitkina and colleagues reformulated a model for filopodia formation proposed previously (Small, 1988, Small et al., 1998) that included the convergence of lamellipodial filaments into filopodia (Svitkina et al., 2003). However, if filopodia were effectively and continuously initiated by the elongation of pre-existing lamellipodial filaments, why would we need actin filament nucleators operating in filopodia such as formins? Furthermore, if the convergent elongation model was 
physiologically relevant, one would expect an interference with the process by removal of lamellipodia, as previously proposed (Biyasheva et al., 2004). Although a more recent study has reaffirmed this conclusion in neurons (Korobova and Svitkina, 2008), several independent studies have continuously failed to establish a defect in filopodia initiation and protrusion effected by suppression of lamellipodia (Gomez et al., 2007, Nicholson-Dykstra and Higgs, 2008, Nobes and Hall, 1995, Sarmiento et al., 2008, Steffen et al., 2006, Vidali et al., 2006). These and similar conflicting observations have prompted researchers to propose different "types” of filopodia, or distinct, cell-type-dependent mechanisms of filopodium formation (see e.g. Gupton and Gertler, 2007). In the absence of experimental evidence for such a scenario, we instead favour the concept of a core assembly machinery, representing essential biochemical activities (see below), and thus a common molecular mechanism that is engaged by divergent upstream signalling pathways (see also Ladwein and Rottner, 2008).

In addition, recent findings obtained with a constitutively active variant of mDia2 (see also below) proved de novo nucleation of actin filaments in filopodia tips (Block et al., 2008), in line with previous observations (Yang et al., 2007). The shape, kinetics and last, not least occasionally unusual initiation sites of these filopodia at the cell periphery - as for instance along the length of pre-existing filopodia - precluded their generation through convergent elongation of lamellipodial filaments (Block et al., 2008).

In spite of the frequently observed co-existence of lamellipodia and filopodia and their apparent interdependence (Small et al., 2002, Svitkina et al., 2003), we propose both distinct signalling pathways and separable core machineries driving the formation of lamellipodia and filopodia (see also Faix and Rottner, 2006). In such a scenario, initiation and continuous protrusion of a filopodium would be driven by de novo nucleation of filopodial filaments, e.g. by a formin.

\section{Formins}


Formins are a group of ubiquitous multi-domain proteins that catalyse the nucleation and elongation of linear actin filaments by insertional assembly of monomers to the fast growing barbed ends. The proline-rich formin homology domain 1 (FH1) recruits profilin-actin complexes for processive filament elongation mediated by the neighbouring $\mathrm{FH} 2$ domain (Kovar et al., 2006, Romero et al., 2004). A subfamily of formins referred to as Diaphanousrelated formins (Drfs) greatly increases the signalling complexity of formin proteins due to their ability to act as effectors of Rho family GTPases (Faix and Grosse, 2006, Goode and Eck, 2007, Higgs, 2005, Watanabe et al., 1997). Based on a phylogenetic analysis, the Drfs can be grouped into three subfamilies: Dia (Diaphanous), Daam (Dishevelled-associated activator of morphogenesis) and FRL (formin-related gene in leukocytes) (Higgs and Peterson, 2005). In the basal state, Drfs exist as auto-inhibited proteins mediated through intramolecular interactions between their $\mathrm{N}$ - and C-terminal regulatory domains (Alberts, 2001, Li and Higgs, 2005). Binding of Rho-GTPases to the regulatory N-terminus harbouring the GTPase-binding domain (GBD) mediates release of auto-inhibition (Nezami et al., 2006, Wallar et al., 2006), and is thought to be the prerequisite for proper subcellular localization of the activated formin (Block et al., 2008, Seth et al., 2006). The FH2 dimer promotes nucleation, and due to its tight interaction with filament barbed ends, subsequently acts as a 'processive capper' (Goode and Eck, 2007, Pruyne et al., 2002, Zigmond et al., 2003) capable of elongating individual filaments hundreds or even thousands of subunits. Interestingly, as compared to other formins, mDia2/Drf3 is a rather slow filament elongator (Kovar et al., 2006). The biochemical characterization of an increasing number of formins strengthens the idea that poor filament elongators are better filament nucleators and vice versa (Neidt et al., 2008), suggesting that some formins such as mDia2 operating in filopodia formation might predominantly drive nucleation rather than elongation (see Table 1). Whether these formins stay constantly associated with the barbed end of the filament during filopodium protrusion or 
if the filament is rapidly released after nucleation and handed over to other elongators (e.g. VASP, see below) is currently unclear.

In Dictyostelium cells, the Drf dDia2 was shown to be critical for filopodium formation suggesting that nucleation and/or elongation of filopodial actin filaments by a formin are key biochemical activities regulating the assembly of these structures (Figure 2) (Schirenbeck et al., 2005). Consistently, GFP-tagged dDia2 was shown to surf on distal tips of protruding filopodia (Schirenbeck et al., 2005). From the fifteen formin isoforms in mammalian cells, so far only two Drfs, referred to as mDia1/Drf1 and mDia2/Drf3, have been implicated in the assembly of filopodial actin filaments (Faix and Grosse, 2006). Constitutively active, Nterminally or C-terminally truncated forms of mDia1 were reported to accumulate at the tips of filopodia-like structures in Xenopus and murine NIH 3T3 fibroblasts, and recently also in MTLn3 rat mammary adenocarcinomas (Copeland et al., 2007, Higashida et al., 2004, Sarmiento et al., 2008). However, the signalling pathway to mDia1-induced filopodia is elusive, since the known interacting GTPases, RhoA, B, and C (Watanabe et al., 1999) have so far not been implicated in filopodia formation (Burridge and Wennerberg, 2004, Faix and Grosse, 2006, Goode and Eck, 2007). Moreover, due to structural constraints, the interaction of mDia1 with the filopodia-inducing GTPase Cdc42 can be excluded (Lammers et al., 2008).

The most prominent formin implicated in filopodia assembly in mammalian cells is the Cdc42- and Rif-effector mDia2/Drf3, since it was reported to localize to their tips after cooverexpression with the active forms of these GTPases (Pellegrin and Mellor, 2005, Peng et al., 2003), and because filopodia formation can be triggered by the expression of constitutively active forms of this formin (Block et al., 2008, Wallar et al., 2006, Yang et al., 2007). As elaborated on above, a novel mDia2/Drf3 variant lacking the C-terminal regulatory region (Drf3 $\triangle \mathrm{DAD}$ ) induced filopodia formation and thickening through de novo nucleation 
(Block et al., 2008). Consistent with previous results obtained with an N-terminally truncated construct (Yang et al., 2007), overexpression of Drf3 $\triangle \mathrm{DAD}$ was shown to promote the formation of numerous, club-shaped filopodia (Block et al., 2008). As assessed by immunofluorescence and electron microscopy, the clubs formed in Drf3 $\triangle \mathrm{DAD}$ overexpressing cells were filled with densely-bundled actin filaments, whose number but not packing density decreased further away from the tip. Moreover, since these filopodia were frequently observed to thicken even after they had separated from the cell periphery, these data demonstrated mDia2-induced filopodia to form and extend by continuous filament nucleation (Block et al., 2008) instead of elongation of a static number of pre-existing filaments as proposed by the 'convergent elongation model' (Svitkina et al., 2003).

The observation that active mDia2 prominently induces filopodia does not yet answer the question of its relevance as compared to other formins potentially expressed in mammalian cells in the process. For instance, some frequently used tissue culture cell models capable of prominent filopodia formation such as NIH and Swiss 3T3 fibroblasts or B16-F1 mouse melanoma cells express minute amounts of the formin (Block et al., 2008, Tominaga et al., 2000). Yet, a recent study suggested loss of both lamellipodia and filopodia upon knockdown of mDia2 in B16-F1 melanomas (Yang et al., 2007), and filopodia were again suggested to derive from Arp2/3- or mDia2-induced lamellipodial filaments. In another conflicting study, mDia2 was instead suppressed by the Arp2/3/WAVE-complex pathway driving lamellipodia formation, but also proposed to function in filopodia formation (Beli et al., 2008). Owing to observations of low expression of mDia2 in certain cell types and the prominent localization of other formins in filopodia tips, a redundant function with other formins is likely.

\section{Ena/VASP proteins}


Initially identified as major substrates for protein kinase $\mathrm{A}$ and $\mathrm{C}$ in platelets (Halbrugge et al., 1990), Ena/VASP proteins subsequently emerged as important regulators of the cytoskeleton. These proteins constitute a structurally conserved family found in vertebrates, invertebrates and motile protozoa such as Dictyostelium amoebae. All members of this family share a tripartite domain architecture: an N-terminal Ena-VASP homology domain 1 (EVH1) required for subcellular localization, a central proline-rich core domain comprising binding sites for profilin as well as for SH3-domain containing proteins, and finally a C-terminal EVH2 domain. The latter contains two WASP homology $\underline{2}$ (WH2) domain-related actin-

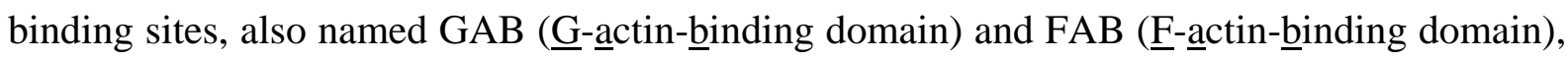
as well as a small coiled-coil region mediating VASP tetramerization (Dominguez, 2007, Ferron et al., 2007, Schirenbeck et al., 2006). Vertebrates express three Ena-related proteins: Mena, Evl and VASP, which were shown to localize to sites of active actin assembly including the surface of intracellular Listeria, focal adhesions, stress fibers, as well as lamellipodial and filopodial tips (Gertler et al., 1996, Lanier et al., 1999, Laurent et al., 1999, Reinhard et al., 1992, Rottner et al., 1999, Svitkina et al., 2003). In agreement with this, Ena/VASP proteins were shown to promote the growth of filopodia in different cell types (Han et al., 2002, Lebrand et al., 2004, Mejillano et al., 2004). Although not essential for filopodium formation in mammals per se (Dent et al., 2007, Mattila and Lappalainen, 2008), the recent elimination of all three Ena/VASP proteins resulted in a markedly reduced formation of filopodia in neuronal cells, and is consistent with the critical function of the single VASP member in Dictyostelium cells (Figure 2) (Dent et al., 2007, Han et al., 2002, Schirenbeck et al., 2006), arguing for a conserved function of Ena/VASP proteins in filopodia formation. Despite these findings, the precise function of these proteins in promoting actin assembly has remained controversial (Trichet et al., 2008). 
Recent experiments employing in vitro TIRF microscopy revealed so far unexpected properties of these proteins. By analyzing single filament dynamics with mammalian and Dictyostelium VASP, it was shown that VASP transiently interacts with filament barbed ends (Breitsprecher et al., 2008, Pasic et al., 2008), and actively drives filament elongation by delivering actin monomers to growing ends in a WH2 domain-dependent fashion (Breitsprecher et al., 2008). Remarkably, VASP clustering on a surface allowed processive filament elongation even in the presence of very high concentrations of heterodimeric capping protein (CP) (Breitsprecher et al., 2008), which may be helpful in driving filament elongation in filopodia. CP resistance is mediated by the FAB motif (or the second WH2 domain). The lack of this feature - and not the absence of filament bundling as initially assumed (Schirenbeck et al., 2006) - presumably explains the inability of FAB-deficient VASP to rescue filopodia formation in Dictyostelium VASP null cells. In summary, this work revealed that VASP is capable of tethering growing filaments to a surface (e.g. the filopodium tip) (Breitsprecher et al., 2008). As opposed to formins that are also able to drive tethered elongation (Romero et al., 2004), Ena/VASP proteins appear unable to nucleate actin filaments in vivo, e.g. on Listeria surfaces (Pistor et al., 2000), or when artificially targeted to mitochondria (Bear et al., 2000). Moreover, fluorescence recovery after photobleaching (FRAP) in B16-F1 melanomas revealed that Ena/VASP proteins rapidly exchange at the leading edge of lamellipodia, whilst almost no exchange occurs at filopodia tips (Applewhite et al., 2007, Lai et al., 2008). Collectively, these data suggest that the key role of VASP clustered in the filopodium tip complex is processive elongation and filament tethering to the cell periphery. It might thus operate in balance to nucleation, accepting filament ends for elongation handed over by the formin. Such a cooperative scenario is supported by evidence for genetic interaction between Dictyostelium VASP and dDia2 (Schirenbeck et al., 2006).

\section{IRSp53}


Besides Ena/VASP family-members, signalling proteins like Abi (Abl interactor) proteins (Stradal et al., 2001) or IRSp53 (Insulin receptor tyrosine kinase substrate of 53kDa) (Nakagawa et al., 2003) were also described to be confined to the very edge or tips of both filopodia and lamellipodia. IRSp53 is the best characterised member of a three gene family in mammals all harbouring an N-terminal IMD (IRSp53/MIM homology Domain) and a more C-terminal SH3 domain. It is still uncertain how exactly these proteins influence the formation of both types of cell protrusions. Although overexpression of IRSp53 family proteins can induce the formation of filopodia or prominent filopodia-like structures, it is not clear if IRSp53 family proteins are essential for filopodium formation per se. To distinguish essential from potential modulatory functions of these proteins in filopodia formation, individual or collective removal of all family members, e.g. using genetics, will be required. It will also be important to define how the IMD of IRSp53 family members contributes to filopodium formation, as multiple activities, like membrane binding, perhaps bending and tubulation or F-actin binding and bundling, have been attributed to this domain (Scita et al., 2008, Suetsugu et al., 2006). Moreover, IRSp53, but not the other family members, harbours a central CRIB (Cdc42/Rac interactive binding) motif rendering it a Cdc42 effector (Yamagishi et al., 2004). In spite of the wealth of information on structure and biological activities of the IMD as well as on potential binding partners of the SH3 domain, the particular molecular activities executed at a given sub-cellular position like lamellipodia or filopodia tips, or its exact interaction partners within the respective tip complexes remain obscure. For example, IRSp53 was suggested to act in Cdc42-dependent filopodium protrusion through its interactions with either the Ena/VASP-family member Mena (Krugmann et al., 2001) or with Eps8 (Disanza et al., 2006), in both cases via its SH3-domain. Both studies proposed that auto-inhibited IRSp53 is activated by these interactions in a Cdc42 dependent fashion, unmasking and/or amplifying the actin-bundling activity of its IMD. However, a more recent 
study questioned a physiological relevance of the actin-bundling activity observed for isolated IMDs at low salt conditions in vitro (Mattila et al., 2007). Furthermore, IRSp53 can also interact with diaphanous-related formins such as mDia1, again via its SH3 domain, but only after the formin is activated by active RhoA (Fujiwara et al., 2000), which operates in retractile and adhesive processes rather than protrusion (Burridge and Wennerberg, 2004). It will thus be instrumental to our understanding of protrusion tip complexes to analyse if IRSp53 interacts with Cdc42-activated formins, e.g. active mDia2/Drf3 at filopodia tips, or how it might be regulated by Ena/VASP interaction.

Overexpression of full length IRSp53 or of isolated IMDs induces filopodium-like membrane protrusions, the majority of which, however, differs significantly from canonical filopodia (Mattila et al., 2007, Millard et al., 2005, Suetsugu et al., 2006). First, ectopically overexpressed IRSp53/IMD localises to the entire plasma membrane enveloping these filopodialike structures (Figure 3), which is different from the localisation pattern of low expressors where IRSp53 is detected in tip complexes of both filopodia (Figure 3) and lamellipodia (compare Disanza et al., 2006, Millard et al., 2005, Nakagawa et al., 2003, Suetsugu et al., 2006). Notably, a convincing localisation of endogenous IRSp53 family porteins in protrusions of motile cells is still lacking, which would be instrumental to resolve the functional relevance of IMD-induced structures. Furthermore, Suetsugu et al. (2006) have concluded that the F-actin content of these protrusions is lower than that of canonical filopodia, in which F-actin is densely packed in parallel bundles (outlined in Figure 3). Furthermore, canonical filopodia harbour at their tips an electron dense protein assembly that was shown to contain actin nucleating and elongating factors (Faix and Rottner, 2006, Svitkina et al., 2003), which is not firmly established for IMD-induced structures. Finally, latrunculin B treatment, which blocks actin polymerisation and consequently filopodium protrusion, did not interfere with tubulation at the cell periphery induced by IMD expression (Suetsugu et al., 2006), thus creating structures resembling filopodium-ghosts rather than 
canonical filopodia. Consistently, mutations of essential basic residues that interfered with membrane tethering also abrogated tubulation (Suetsugu et al., 2006), which is in line with independent analyses of similar mutations that eliminated the known biological activities of IMDs (Millard et al., 2005, Millard et al., 2007).

Together, these data suggest IRSp53-mediated membrane binding and bending to have important physiological consequences for the formation of canonical filopodia, but at the same time to cause exaggerated membrane tubules upon over-expression, which are independent of actin and thus lack a physiological correlate at the protrusive front of migrating cells.

\section{Concluding remarks}

Through the combination of multiple approaches, extending from biochemistry to modern imaging and conditional and multiple gene removal, we have experienced an explosion of information on different molecular activities implicated in filopodia formation (Figure 4). Nevertheless, we are still far from a complete picture of how these dynamic structures are initiated, maintained and disassembled, and which signalling pathways share the capability of inducing them. For instance, we still do not know which of the fifteen mammalian formins can operate in filopodia formation, if some of them may be redundant or have exclusive functions essential for the process. It is also not entirely clear why interference with filament elongation, as effected for instance by removal of Dictyostelium VASP, is so detrimental for filopodium formation, or cannot be compensated for by formins. Additional molecular activities important for filopodium formation are also elusive, such as those exerted by unconventional myosins (Figure 2). Finally, is it possible to define a core, biochemical machinery responsible for filopodium initiation and protrusion, which functions in various cell types and is potentially engaged by divergent signalling pathways? Components of such a 
machinery would be expected to localize to any given filopodium, irrespective of cell type and tissue or pathway of its induction, and to cause at least a moderate phenotype upon functional interference. Both criteria apply to a minimal set of proteins depicted here (Figure 4): First, filopodial regulators such as VASP, formins or myosin $\mathrm{X}$ (the mammalian homologue of Dictyostelium myosin VII; see Figures 2 and 4) are to our knowledge not known to be restricted to a subtype of filopodia in any given cell type or tissue. Second, genetic deletion of members of all these protein families established to accumulate in filopodia tips strongly compromised filopodium formation in Dictyostelium cells (Figure 2). Furthermore, the relevance of bundling of actin filaments in the filopodium shaft has been exemplified by RNA interference-mediated depletion of fascin (Vignjevic et al., 2006). Thus, according to these criteria, it is reasonable to consider the aforementioned components as part of a potential core machinery mediating biochemical activities required for the formation of these structures. Finally, distinct filopodia characteristics such as variable lengths, widths or dynamics could well be caused by the balance of this small set of biochemical activities essential for filopodium formation and turnover. We assume that these activities may be restricted to nucleation, elongation, bundling and disassembly (Figure 4), although direct experimental evidence for the relevance of the latter is currently lacking. Future work will require determination of the relative relevance of all these biochemical activities to explain filopodia formation in different cells and tissues. 


\title{
Acknowledgements
}

We thank Antje K. Kiesewetter (Hannover Medical School) for help with assembling Figure 2. Myosin VII-null cells and the GFP-myosin VII expression vector were kindly provided by Dr. Margaret Titus (University of Minnesota). We are grateful to J. Victor Small (Institute of Molecular Biotechnology, Vienna) for insightful discussion and critical reading of the manuscript. This work was supported by grants from the Deutsche Forschungsgemeinschaft (DFG) to T.E.B.S and K.R. (STR666/2-3) and to J.F. (FA330/4-1).

\begin{abstract}
Abbreviations
Abi: Abl interactor; Arp: actin-related protein; CP: capping protein; CRIB: Cdc42/Rac interactive binding; Drf: Diaphanous-related formin; Ena: enabled; Eps8: epidermal growth factor receptor pathway substrate 8; Evl: Ena/VASP-like; FRAP: fluorescence recovery after photobleaching; IRSp53: Insulin receptor tyrosine kinase substrate of 53kDa; IMD: IRSp53/MIM homology domain; Mena: mammalian enabled; MIM: missing in metastasis; Rif: Rho in filopodia; SH3: Src homology 3; TIRF: total internal reflection fluorescence; VASP: vasodilator stimulated phosphoprotein; WASP: Wiskott-Aldrich syndrome protein; WAVE: WASP family verprolin-homologous; WH2: WASP homology 2; Wrch: Wnt-1 responsive Cdc42 homolog.
\end{abstract}




\section{References}

Adams, J. C. (2001). Cell-matrix contact structures. Cell Mol Life Sci, 58, 371-392.

Alberts, A. S. (2001). Identification of a carboxyl-terminal diaphanous-related formin homology protein autoregulatory domain. J Biol Chem, 276, 2824-2830.

Applewhite, D. A., Barzik, M., Kojima, S., Svitkina, T. M., Gertler, F. B. \& Borisy, G. G. (2007). Ena/VASP proteins have an anti-capping independent function in filopodia formation. Mol Biol Cell, 18, 2579-2591.

Aspenstrom, P., Fransson, A. \& Saras, J. (2004). Rho GTPases have diverse effects on the organization of the actin filament system. Biochem J, 377, 327-337.

Ballestrem, C., Wehrle-Haller, B. \& Imhof, B. A. (1998). Actin dynamics in living mammalian cells. J Cell Sci, 111 ( Pt 12), 1649-1658.

Bear, J. E., Loureiro, J. J., Libova, I., Fassler, R., Wehland, J. \& Gertler, F. B. (2000). Negative regulation of fibroblast motility by Ena/VASP proteins. Cell, 101, 717-728.

Beli, P., Mascheroni, D., Xu, D. \& Innocenti, M. (2008). WAVE and Arp2/3 jointly inhibit filopodium formation by entering into a complex with mDia2. Nat Cell Biol, 10, 849857.

Biyasheva, A., Svitkina, T., Kunda, P., Baum, B. \& Borisy, G. (2004). Cascade pathway of filopodia formation downstream of SCAR. J Cell Sci, 117, 837-848.

Block, J., Stradal, T. E., Hanisch, J., Geffers, R., Kostler, S. A., Urban, E., Small, J. V., Rottner, K. \& Faix, J. (2008). Filopodia formation induced by active mDia2/Drf3. $J$ Microsc, 231, 506-517.

Breitsprecher, D., Kiesewetter, A. K., Linkner, J., Urbanke, C., Resch, G. P., Small, J. V. \& Faix, J. (2008). Clustering of VASP actively drives processive, WH2 domainmediated actin filament elongation. Embo $J$.

Burridge, K. \& Wennerberg, K. (2004). Rho and Rac take center stage. Cell, 116, 167-179.

Copeland, S. J., Green, B. J., Burchat, S., Papalia, G. A., Banner, D. \& Copeland, J. W. (2007). The diaphanous inhibitory domain/diaphanous autoregulatory domain interaction is able to mediate heterodimerization between mDia1 and mDia2. J Biol Chem, 282, 30120-30130.

Czuchra, A., Wu, X., Meyer, H., van Hengel, J., Schroeder, T., Geffers, R., Rottner, K. \& Brakebusch, C. (2005). Cdc42 is not essential for filopodium formation, directed migration, cell polarization, and mitosis in fibroblastoid cells. Mol Biol Cell, 16, 44734484.

Dent, E. W., Kwiatkowski, A. V., Mebane, L. M., Philippar, U., Barzik, M., Rubinson, D. A., Gupton, S., Van Veen, J. E., Furman, C., Zhang, J., Alberts, A. S., Mori, S. \& Gertler, F. B. (2007). Filopodia are required for cortical neurite initiation. Nat Cell Biol, 9, 1347-1359.

DerMardirossian, C. \& Bokoch, G. M. (2005). GDIs: central regulatory molecules in Rho GTPase activation. Trends Cell Biol, 15, 356-363.

Disanza, A., Mantoani, S., Hertzog, M., Gerboth, S., Frittoli, E., Steffen, A., Berhoerster, K., Kreienkamp, H. J., Milanesi, F., Di Fiore, P. P., Ciliberto, A., Stradal, T. E. \& Scita, G. (2006). Regulation of cell shape by Cdc42 is mediated by the synergic actinbundling activity of the Eps8-IRSp53 complex. Nat Cell Biol, 8, 1337-1347.

Disanza, A., Steffen, A., Hertzog, M., Frittoli, E., Rottner, K. \& Scita, G. (2005). Actin polymerization machinery: the finish line of signaling networks, the starting point of cellular movement. Cell Mol Life Sci, 62, 955-970.

Dominguez, R. (2007). The beta-thymosin/WH2 fold: multifunctionality and structure. Ann $N$ Y Acad Sci, 1112, 86-94. 
Dumontier, M., Hocht, P., Mintert, U. \& Faix, J. (2000). Rac1 GTPases control filopodia formation, cell motility, endocytosis, cytokinesis and development in Dictyostelium. $J$ Cell Sci, 113 ( Pt 12), 2253-2265.

Ellis, S. \& Mellor, H. (2000). The novel Rho-family GTPase rif regulates coordinated actinbased membrane rearrangements. Curr Biol, 10, 1387-1390.

Faix, J. \& Grosse, R. (2006). Staying in shape with formins. Dev Cell, 10, 693-706.

Faix, J. \& Rottner, K. (2006). The making of filopodia. Curr Opin Cell Biol, 18, 18-25.

Ferron, F., Rebowski, G., Lee, S. H. \& Dominguez, R. (2007). Structural basis for the recruitment of profilin-actin complexes during filament elongation by Ena/VASP. Embo J, 26, 4597-4606.

Fujiwara, T., Mammoto, A., Kim, Y. \& Takai, Y. (2000). Rho small G-protein-dependent binding of mDia to an Src homology 3 domain-containing IRSp53/BAIAP2. Biochem Biophys Res Commun, 271, 626-629.

Gertler, F. B., Niebuhr, K., Reinhard, M., Wehland, J. \& Soriano, P. (1996). Mena, a relative of VASP and Drosophila Enabled, is implicated in the control of microfilament dynamics. Cell, 87, 227-239.

Gomez, T. S., Kumar, K., Medeiros, R. B., Shimizu, Y., Leibson, P. J. \& Billadeau, D. D. (2007). Formins regulate the actin-related protein 2/3 complex-independent polarization of the centrosome to the immunological synapse. Immunity, 26, 177-190.

Goode, B. L. \& Eck, M. J. (2007). Mechanism and function of formins in the control of actin assembly. Annu Rev Biochem, 76, 593-627.

Gupton, S. L. \& Gertler, F. B. (2007). Filopodia: the fingers that do the walking. Sci STKE, 2007, re5.

Halbrugge, M., Friedrich, C., Eigenthaler, M., Schanzenbacher, P. \& Walter, U. (1990). Stoichiometric and reversible phosphorylation of a $46-\mathrm{kDa}$ protein in human platelets in response to cGMP- and cAMP-elevating vasodilators. J Biol Chem, 265, 30883093.

Han, Y. H., Chung, C. Y., Wessels, D., Stephens, S., Titus, M. A., Soll, D. R. \& Firtel, R. A. (2002). Requirement of a vasodilator-stimulated phosphoprotein family member for cell adhesion, the formation of filopodia, and chemotaxis in dictyostelium. $\mathrm{J}$ Biol Chem, 277, 49877-49887.

Higashida, C., Miyoshi, T., Fujita, A., Oceguera-Yanez, F., Monypenny, J., Andou, Y., Narumiya, S. \& Watanabe, N. (2004). Actin polymerization-driven molecular movement of mDia1 in living cells. Science, 303, 2007-2010.

Higgs, H. N. (2005). Formin proteins: a domain-based approach. Trends Biochem Sci, 30, 342-353.

Higgs, H. N. \& Peterson, K. J. (2005). Phylogenetic analysis of the formin homology 2 domain. Mol Biol Cell, 16, 1-13.

Koestler, S. A., Auinger, S., Vinzenz, M., Rottner, K. \& Small, J. V. (2008). Differentially oriented populations of actin filaments generated in lamellipodia collaborate in pushing and pausing at the cell front. Nat Cell Biol, 10, 306-313.

Korobova, F. \& Svitkina, T. (2008). Arp2/3 complex is important for filopodia formation, growth cone motility, and neuritogenesis in neuronal cells. Mol Biol Cell, 19, 15611574.

Kovar, D. R., Harris, E. S., Mahaffy, R., Higgs, H. N. \& Pollard, T. D. (2006). Control of the assembly of ATP- and ADP-actin by formins and profilin. Cell, 124, 423-435.

Kozma, R., Ahmed, S., Best, A. \& Lim, L. (1995). The Ras-related protein Cdc42Hs and bradykinin promote formation of peripheral actin microspikes and filopodia in Swiss 3 T3 fibroblasts. Mol Cell Biol, 15, 1942-1952. 
Krugmann, S., Jordens, I., Gevaert, K., Driessens, M., Vandekerckhove, J. \& Hall, A. (2001). Cdc42 induces filopodia by promoting the formation of an IRSp53:Mena complex. Curr Biol, 11, 1645-1655.

Ladwein, M. \& Rottner, K. (2008). On the Rho'd: the regulation of membrane protrusions by Rho-GTPases. FEBS Lett, 582, 2066-2074.

Lai, F. P., Szczodrak, M., Block, J., Faix, J., Breitsprecher, D., Mannherz, H. G., Stradal, T. E., Dunn, G. A., Small, J. V. \& Rottner, K. (2008). Arp2/3 complex interactions and actin network turnover in lamellipodia. Embo J, 27, 982-992.

Lambrechts, A., Gevaert, K., Cossart, P., Vandekerckhove, J. \& Van Troys, M. (2008). Listeria comet tails: the actin-based motility machinery at work. Trends Cell Biol, 18, 220-227.

Lammers, M., Meyer, S., Kuhlmann, D. \& Wittinghofer, A. (2008). The specificity of interactions between mdia-isoforms and rho proteins. J Biol Chem.

Lanier, L. M., Gates, M. A., Witke, W., Menzies, A. S., Wehman, A. M., Macklis, J. D., Kwiatkowski, D., Soriano, P. \& Gertler, F. B. (1999). Mena is required for neurulation and commissure formation. Neuron, 22, 313-325.

Laurent, V., Loisel, T. P., Harbeck, B., Wehman, A., Grobe, L., Jockusch, B. M., Wehland, J., Gertler, F. B. \& Carlier, M. F. (1999). Role of proteins of the Ena/VASP family in actin-based motility of Listeria monocytogenes. J Cell Biol, 144, 1245-1258.

Lebrand, C., Dent, E. W., Strasser, G. A., Lanier, L. M., Krause, M., Svitkina, T. M., Borisy, G. G. \& Gertler, F. B. (2004). Critical role of Ena/VASP proteins for filopodia formation in neurons and in function downstream of netrin-1. Neuron, 42, 37-49.

Li, F. \& Higgs, H. N. (2005). Dissecting requirements for auto-inhibition of actin nucleation by the formin, mDia1. J Biol Chem, 280, 6986-6992.

Lommel, S., Benesch, S., Rottner, K., Franz, T., Wehland, J. \& Kuhn, R. (2001). Actin pedestal formation by enteropathogenic Escherichia coli and intracellular motility of Shigella flexneri are abolished in N-WASP-defective cells. EMBO Rep, 2, 850-857.

Mallavarapu, A. \& Mitchison, T. (1999). Regulated actin cytoskeleton assembly at filopodium tips controls their extension and retraction. J Cell Biol, 146, 1097-1106.

Mattila, P. K. \& Lappalainen, P. (2008). Filopodia: molecular architecture and cellular functions. Nat Rev Mol Cell Biol, 9, 446-454.

Mattila, P. K., Pykalainen, A., Saarikangas, J., Paavilainen, V. O., Vihinen, H., Jokitalo, E. \& Lappalainen, P. (2007). Missing-in-metastasis and IRSp53 deform PI(4,5)P2-rich membranes by an inverse BAR domain-like mechanism. J Cell Biol, 176, 953-964.

Mejillano, M. R., Kojima, S., Applewhite, D. A., Gertler, F. B., Svitkina, T. M. \& Borisy, G. G. (2004). Lamellipodial versus filopodial mode of the actin nanomachinery: pivotal role of the filament barbed end. Cell, 118, 363-373.

Mellor, H. (2009). The role of formins in filopodia formation. Biochim Biophys Acta.

Millard, T. H., Bompard, G., Heung, M. Y., Dafforn, T. R., Scott, D. J., Machesky, L. M. \& Futterer, K. (2005). Structural basis of filopodia formation induced by the IRSp53/MIM homology domain of human IRSp53. Embo J, 24, 240-250.

Millard, T. H., Dawson, J. \& Machesky, L. M. (2007). Characterisation of IRTKS, a novel IRSp53/MIM family actin regulator with distinct filament bundling properties. J Cell Sci, 120, 1663-1672.

Moon, S. Y. \& Zheng, Y. (2003). Rho GTPase-activating proteins in cell regulation. Trends Cell Biol, 13, 13-22.

Nakagawa, H., Miki, H., Nozumi, M., Takenawa, T., Miyamoto, S., Wehland, J. \& Small, J. V. (2003). IRSp53 is colocalised with WAVE2 at the tips of protruding lamellipodia and filopodia independently of Mena. J Cell Sci, 116, 2577-2583. 
Neidt, E. M., Skau, C. T. \& Kovar, D. R. (2008). The cytokinesis formins from the nematode worm and fission yeast differentially mediate actin filament assembly. $\mathrm{J}$ Biol Chem, 283, 23872-23883.

Nezami, A. G., Poy, F. \& Eck, M. J. (2006). Structure of the autoinhibitory switch in formin mDia1. Structure, 14, 257-263.

Nicholson-Dykstra, S. M. \& Higgs, H. N. (2008). Arp2 depletion inhibits sheet-like protrusions but not linear protrusions of fibroblasts and lymphocytes. Cell Motil Cytoskeleton, 65, 904-922.

Nobes, C. D. \& Hall, A. (1995). Rho, rac, and cdc42 GTPases regulate the assembly of multimolecular focal complexes associated with actin stress fibers, lamellipodia, and filopodia. Cell, 81, 53-62.

Oldenbourg, R., Katoh, K. \& Danuser, G. (2000). Mechanism of lateral movement of filopodia and radial actin bundles across neuronal growth cones. Biophys $J, 78,1176-$ 1182.

Pasic, L., Kotova, T. \& Schafer, D. A. (2008). Ena/VASP proteins capture actin filament barbed ends. J Biol Chem, 283, 9814-9819.

Pellegrin, S. \& Mellor, H. (2005). The Rho family GTPase Rif induces filopodia through mDia2. Curr Biol, 15, 129-133.

Peng, J., Wallar, B. J., Flanders, A., Swiatek, P. J. \& Alberts, A. S. (2003). Disruption of the Diaphanous-related formin Drf1 gene encoding mDia1 reveals a role for Drf3 as an effector for Cdc42. Curr Biol, 13, 534-545.

Pistor, S., Grobe, L., Sechi, A. S., Domann, E., Gerstel, B., Machesky, L. M., Chakraborty, T. \& Wehland, J. (2000). Mutations of arginine residues within the 146-KKRRK-150 motif of the ActA protein of Listeria monocytogenes abolish intracellular motility by interfering with the recruitment of the Arp2/3 complex. J Cell Sci, 113 ( Pt 18), 32773287.

Pollard, T. D. \& Borisy, G. G. (2003). Cellular motility driven by assembly and disassembly of actin filaments. Cell, 112, 453-465.

Pruyne, D., Evangelista, M., Yang, C., Bi, E., Zigmond, S., Bretscher, A. \& Boone, C. (2002). Role of formins in actin assembly: nucleation and barbed-end association. Science, 297, 612-615.

Reinhard, M., Halbrugge, M., Scheer, U., Wiegand, C., Jockusch, B. M. \& Walter, U. (1992). The 46/50 kDa phosphoprotein VASP purified from human platelets is a novel protein associated with actin filaments and focal contacts. Embo J, 11, 2063-2070.

Romero, S., Le Clainche, C., Didry, D., Egile, C., Pantaloni, D. \& Carlier, M. F. (2004). Formin is a processive motor that requires profilin to accelerate actin assembly and associated ATP hydrolysis. Cell, 119, 419-429.

Rossman, K. L., Der, C. J. \& Sondek, J. (2005). GEF means go: turning on RHO GTPases with guanine nucleotide-exchange factors. Nat Rev Mol Cell Biol, 6, 167-180.

Rottner, K., Behrendt, B., Small, J. V. \& Wehland, J. (1999). VASP dynamics during lamellipodia protrusion. Nat Cell Biol, 1, 321-322.

Ruusala, A. \& Aspenstrom, P. (2008). The atypical Rho GTPase Wrch1 collaborates with the nonreceptor tyrosine kinases Pyk2 and Src in regulating cytoskeletal dynamics. Mol Cell Biol, 28, 1802-1814.

Sarmiento, C., Wang, W., Dovas, A., Yamaguchi, H., Sidani, M., El-Sibai, M., Desmarais, V., Holman, H. A., Kitchen, S., Backer, J. M., Alberts, A. \& Condeelis, J. (2008). WASP family members and formin proteins coordinate regulation of cell protrusions in carcinoma cells. J Cell Biol, 180, 1245-1260.

Schirenbeck, A., Arasada, R., Bretschneider, T., Stradal, T. E., Schleicher, M. \& Faix, J. (2006). The bundling activity of vasodilator-stimulated phosphoprotein is required for filopodium formation. Proc Natl Acad Sci U S A, 103, 7694-7699. 
Schirenbeck, A., Bretschneider, T., Arasada, R., Schleicher, M. \& Faix, J. (2005). The Diaphanous-related formin dDia2 is required for the formation and maintenance of filopodia. Nat Cell Biol, 7, 619-625.

Scita, G., Confalonieri, S., Lappalainen, P. \& Suetsugu, S. (2008). IRSp53: crossing the road of membrane and actin dynamics in the formation of membrane protrusions. Trends Cell Biol, 18, 52-60.

Seth, A., Otomo, C. \& Rosen, M. K. (2006). Autoinhibition regulates cellular localization and actin assembly activity of the diaphanous-related formins FRLalpha and mDia1. J Cell Biol, 174, 701-713.

Small, J. V. (1988). The actin cytoskeleton. Electron Microsc Rev, 1, 155-174.

Small, J. V., Rottner, K., Kaverina, I. \& Anderson, K. I. (1998). Assembling an actin cytoskeleton for cell attachment and movement. Biochim Biophys Acta, 1404, 271281.

Small, J. V., Stradal, T., Vignal, E. \& Rottner, K. (2002). The lamellipodium: where motility begins. Trends Cell Biol, 12, 112-120.

Snapper, S. B., Takeshima, F., Anton, I., Liu, C. H., Thomas, S. M., Nguyen, D., Dudley, D., Fraser, H., Purich, D., Lopez-Ilasaca, M., Klein, C., Davidson, L., Bronson, R., Mulligan, R. C., Southwick, F., Geha, R., Goldberg, M. B., Rosen, F. S., Hartwig, J. H. \& Alt, F. W. (2001). N-WASP deficiency reveals distinct pathways for cell surface projections and microbial actin-based motility. Nat Cell Biol, 3, 897-904.

Somesh, B. P., Vlahou, G., Iijima, M., Insall, R. H., Devreotes, P. \& Rivero, F. (2006). RacG regulates morphology, phagocytosis, and chemotaxis. Eukaryot Cell, 5, 1648-1663.

Steffen, A., Faix, J., Resch, G. P., Linkner, J., Wehland, J., Small, J. V., Rottner, K. \& Stradal, T. E. (2006). Filopodia formation in the absence of functional WAVE- and Arp2/3-complexes. Mol Biol Cell, 17, 2581-2591.

Stevens, J. M., Galyov, E. E. \& Stevens, M. P. (2006). Actin-dependent movement of bacterial pathogens. Nat Rev Microbiol, 4, 91-101.

Stradal, T., Courtney, K. D., Rottner, K., Hahne, P., Small, J. V. \& Pendergast, A. M. (2001). The Abl interactor proteins localize to sites of actin polymerization at the tips of lamellipodia and filopodia. Curr Biol, 11, 891-895.

Stradal, T. E., Rottner, K., Disanza, A., Confalonieri, S., Innocenti, M. \& Scita, G. (2004). Regulation of actin dynamics by WASP and WAVE family proteins. Trends Cell Biol, 14, 303-311.

Suetsugu, S., Murayama, K., Sakamoto, A., Hanawa-Suetsugu, K., Seto, A., Oikawa, T., Mishima, C., Shirouzu, M., Takenawa, T. \& Yokoyama, S. (2006). The RAC binding domain/IRSp53-MIM homology domain of IRSp53 induces RAC-dependent membrane deformation. J Biol Chem, 281, 35347-35358.

Svitkina, T. M. \& Borisy, G. G. (1999). Arp2/3 complex and actin depolymerizing factor/cofilin in dendritic organization and treadmilling of actin filament array in lamellipodia. J Cell Biol, 145, 1009-1026.

Svitkina, T. M., Bulanova, E. A., Chaga, O. Y., Vignjevic, D. M., Kojima, S., Vasiliev, J. M. \& Borisy, G. G. (2003). Mechanism of filopodia initiation by reorganization of a dendritic network. J Cell Biol, 160, 409-421.

Tominaga, T., Sahai, E., Chardin, P., McCormick, F., Courtneidge, S. A. \& Alberts, A. S. (2000). Diaphanous-related formins bridge Rho GTPase and Src tyrosine kinase signaling. Mol Cell, 5, 13-25.

Trichet, L., Sykes, C. \& Plastino, J. (2008). Relaxing the actin cytoskeleton for adhesion and movement with Ena/VASP. J Cell Biol, 181, 19-25.

Vidali, L., Chen, F., Cicchetti, G., Ohta, Y. \& Kwiatkowski, D. J. (2006). Rac1-null mouse embryonic fibroblasts are motile and respond to platelet-derived growth factor. $\mathrm{Mol}$ Biol Cell, 17, 2377-2390. 
Vignjevic, D., Kojima, S., Aratyn, Y., Danciu, O., Svitkina, T. \& Borisy, G. G. (2006). Role of fascin in filopodial protrusion. J Cell Biol, 174, 863-875.

Wallar, B. J., Stropich, B. N., Schoenherr, J. A., Holman, H. A., Kitchen, S. M. \& Alberts, A. S. (2006). The basic region of the diaphanous-autoregulatory domain (DAD) is required for autoregulatory interactions with the diaphanous-related formin inhibitory domain. J Biol Chem, 281, 4300-4307.

Wang, Y. L. (1985). Exchange of actin subunits at the leading edge of living fibroblasts: possible role of treadmilling. J Cell Biol, 101, 597-602.

Watanabe, N., Kato, T., Fujita, A., Ishizaki, T. \& Narumiya, S. (1999). Cooperation between mDia1 and ROCK in Rho-induced actin reorganization. Nat Cell Biol, 1, 136-143.

Watanabe, N., Madaule, P., Reid, T., Ishizaki, T., Watanabe, G., Kakizuka, A., Saito, Y., Nakao, K., Jockusch, B. M. \& Narumiya, S. (1997). p140mDia, a mammalian homolog of Drosophila diaphanous, is a target protein for Rho small GTPase and is a ligand for profilin. Embo J, 16, 3044-3056.

Yamagishi, A., Masuda, M., Ohki, T., Onishi, H. \& Mochizuki, N. (2004). A novel actin bundling/filopodium-forming domain conserved in insulin receptor tyrosine kinase substrate p53 and missing in metastasis protein. J Biol Chem, 279, 14929-14936.

Yang, C., Czech, L., Gerboth, S., Kojima, S., Scita, G. \& Svitkina, T. (2007). Novel roles of formin mDia2 in lamellipodia and filopodia formation in motile cells. PLoS Biol, 5, e317.

Yang, L., Wang, L. \& Zheng, Y. (2006). Gene targeting of Cdc42 and Cdc42GAP affirms the critical involvement of Cdc42 in filopodia induction, directed migration, and proliferation in primary mouse embryonic fibroblasts. Mol Biol Cell, 17, 4675-4685.

Zigmond, S. H., Evangelista, M., Boone, C., Yang, C., Dar, A. C., Sicheri, F., Forkey, J. \& Pring, M. (2003). Formin leaky cap allows elongation in the presence of tight capping proteins. Curr Biol, 13, 1820-1823. 


\section{Table and Figure legends}

Table 1. Comparison of different formin activities. Biochemical properties of selected formins show a direct correlation between their elongation and dissociation rates in the presence of profilin. Published values for the nucleation efficiencies of S. pombe Cdc12 and C. elegans CYK-1 further suggest that fast elongators are poor nucleators and vice versa $\left({ }^{2}\right.$ Kovar et al., 2006, ${ }^{1}$ Neidt et al., 2008). In keeping with this, the poor elongation rate obtained for mDia2 might indicate strong nucleation activity (as opposed to mDia1), which is consistent with mDia2-induced, club-shaped filopodia in vivo (Block et al., 2008, Yang et al., 2007), but remains to be experimentally confirmed in vitro.

Fig. 1. Organization of cellular F-actin structures and filopodia in different cell types. (A) Overview of a schematized cell depicting distinct F-actin-containing structures. Microspikes and filopodia contain bundles of parallel actin filaments with the barbed ends facing towards the cell periphery. (B) Filopodia are found on the surfaces of different cell types. Despite considerable variation in overall cell morphology, the different cell types and/or tissues all express filopodia to various extents, the structural organization of which is highly conserved. The filopodia found in Dictyostelium cells appear more flexible as compared to most mammalian counterparts, which might be due to the absence of the actin-bundling protein fascin.

Fig. 2. Important players of filopodium formation in Dictyostelium cells as revealed by genetic deletion and rescue. (A, C, D) F-actin organization and ability of cells to form filopodia in the wild type, in mutants lacking either VASP (Han et al., 2002), the formin dDia2 (Schirenbeck et al., 2005) or the myosin X-related myosin VII (Tuxworth et al., 2001), and in respective reconstituted cells lines (Rescue). Cells were fixed and stained with 
phalloidin to visualize F-actin alone or F-actin (red) and the respective GFP-tagged filopodial regulator (green). Note their accumulation at the tips (dDia2) or tip regions (myosin VII) of filopodial actin bundles. (B) Single confocal section of a time lapse series of a VASP-null cell expressing GFP-tagged VASP illustrating the transient accumulation of VASP in the tip region of a protruding pseudopod (white triangle) - the equivalent to lamellipodia in mammalian cells - and in the tips of filopodia (white asterisk). Scale bars are 10 and $5 \mu \mathrm{m}$ in grey level and coloured images, respectively.

Fig. 3. Model of filopodium (A) and filopodium-like membrane evagination (B) as induced by IRSp53 expression. Due to significant differences in appearance and dynamics of these two types of structures (for details see text), we suggest to term IMD-induced membrane tubules at the cell periphery filopodia-like structures or ghosts rather than filopodia.

Fig. 4. Core activities required for filopodium initiation and protrusion. Filopodium formation requires a balance of biochemical activities that are summarised here. These activities include nucleation of actin filaments, as induced for instance by formins, their elongation and concomitant tethering to membranes, potentially mediated by various factors such as formins or VASP, bundling or cross-linking represented by bundling proteins like fascin, espin or fimbrin (Vignjevic et al., 2006), and perhaps myosin $\mathrm{X}$ at filopodia tips, and their disassembly. The latter could be accomplished by cofilin or complexes containing additional factors (Kueh et al., 2008). We propose the ultrastructure of filopodia to be modulated by these core biochemical activities, which are all operating simultaneously, and in a balanced fashion during continuous protrusion of filopodia. Thus, increased nucleation as compared to the rest of all activities triggered by an active formin variant (Block et al., 2008) leads to clubshaped filopodia containing excess filaments in the tip, whereas reduced nucleation/elongation impairs the process (see also Figure 2). In contrast, increased elongation 
would be expected to cause tapering of filopodia, which remains to be experimentally verified (?), at least assuming that lost filaments are continuously substituted by de novo nucleated ones. 